\title{
Precipitation Nowcasting using Data-driven Reduced-order Model
}

\author{
Divya S Vidyadharan, Aaron Xavier, Blossom Treesa Bastian, Ajay Ragh and Naveen Francis Chittilapilly
}

\begin{abstract}
Radar-based precipitation nowcasting refers to predicting rain for a short period of time using radar reflectivity images. For dynamic nowcasting, motion fields can be extrapolated using an approximate and localized reduced-order model. Motion field estimation based on traditional Horn-Schunck (HS) algorithm suffers from over-smoothing at discontinuities in nonrigid and dissolving texture present in precipitation nowcasting products. An attempt to preserve the discontinuities using an L1 norm formulation in HS led to the use of Total Variation L1 norm (TVL1). In this paper, we propose a radar-based precipitation nowcasting model with TVL1-based estimation of motion field and Sparse Identification of Non-linear Dynamics (SINDy)-based estimation of non-linear dynamics. TVL1 is effective in preserving the edges especially in the case of the eye of typhoons and squall lines while estimating motion vectors. SINDy captures the nonlinear dynamics and generates the subsequent update values for the motion field based on a reduced-order representation. Finally, the SINDy-generated ensemble of motion field is used along with the radar reflectivity image for generating precipitation nowcasts. We evaluated the effectiveness of TVL1 in preserving edges while capturing the motion field from non-rigid surfaces. The performance of the proposed TVL1-SINDy model in nowcasting weather events such as Typhoons and Squall lines are evaluated using performance metrics such as Mean Absolute Error (MAE), and Critical Success Index (CSI). Experimental results show that the proposed nowcasting system demonstrates better performance compared to the benchmark nowcasting models with lower MAE, higher CSI at higher lead times.
\end{abstract}

Index Terms-Radar Precipitation Nowcasting, TVL1, optical flow, SINDy.

\section{INTRODUCTION}

$\mathbf{N}$ OWCASTING encompasses a wide range of techniques for weather forecasting with short lead time in the near future (0-6 hours) based on high-resolution observations from remote sensing instruments including radars [1] and satellites [2], [3]. Several prior works have shown that nowcasting performs better than Numerical Weather Prediction (NWP) models for short lead time (0-6 hr) [4], [5], [6]. High impact weather events including convective storms and cyclonic storms can create unexpected havoc in our social life and economical preferences. Heavy convective storms and cyclones can cause flash flooding, flooding in coastal areas, and derail the transportation both on land and air. Precipitation nowcasting has gained significant research interest in recent years. At a time when the global climate crisis resulting in flash floods and extreme weather events in an unprecedented

D. S. Vidyadharan, A. Xavier, B. T. Bastian, A. Ragh are with Augsense Lab, India e-mail:divya, aaron, blossom, ajayragh@augsenselab.com.

N. F. Chittilapilly is with ACSL Ltd., Japan e-mail:chittilapilly@acsl.co.jp. (Corresponding author: Divya S Vidyadharan)

Manuscript received xxxx xx, 2020; revised xxxx xx, 2020.

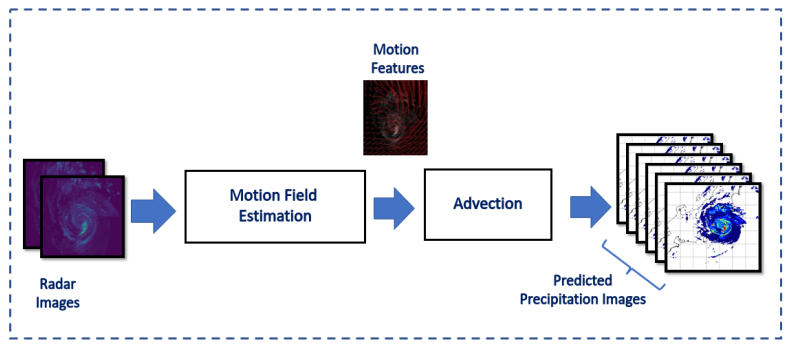

Fig. 1: Different phases of radar precipitation nowcasting. A typical nowcasting model consisting of motion field tracking and advection.

manner, an accurate nowcasting system can facilitate local governments to make better decisions regarding evacuation plans, and optimization of travel routes.

Weather phenomena are influenced by many known natural factors and unknown atmospheric dynamics that are often hard to model and lead to uncertainty in prediction. Rainy storms exhibit extreme variability in their pattern. The time evolution of precipitation is affected by various environmental factors including the nature of the terrain, the presence of atmospheric low-pressure spots, and the Coriolis force. Researchers resort to several mathematical, physical, and data-driven techniques to understand weather events and model them.

Each precipitation event usually exhibits persistence over a period depending on the nature of the event. Quantitative precipitation nowcasting is categorized into climatological persistence, Eulerian persistence, and Lagrangian Persistence [7], [8], [9] based on how the persistence of weather phenomena is considered. In climatological persistence, the prediction is based on the mean or median of climatological values. In Eulerian persistence, the recent precipitation image is taken as the prediction. This is simple, yet an effective measure to compare the skill of a nowcast system. Lagrangian persistence advects the recent radar precipitation using a motion field assuming precipitation data remains constant.

Lagrangian persistence-based nowcasting consists of a tracking step followed by an advection phase. In the tracking phase, the initial step is to estimate the motion or velocity field of precipitation. Tracking methods are classified as cell tracking, and area tracking based on the level at which the motion field is estimated. In cell tracking, motion vectors at each cell/pixel/grid are computed whereas, in area tracking, the motion vectors of continuous objects/regions are tracked. In this paper, we propose a nowcasting system that estimates motion field of precipitation with fine details and that considers 


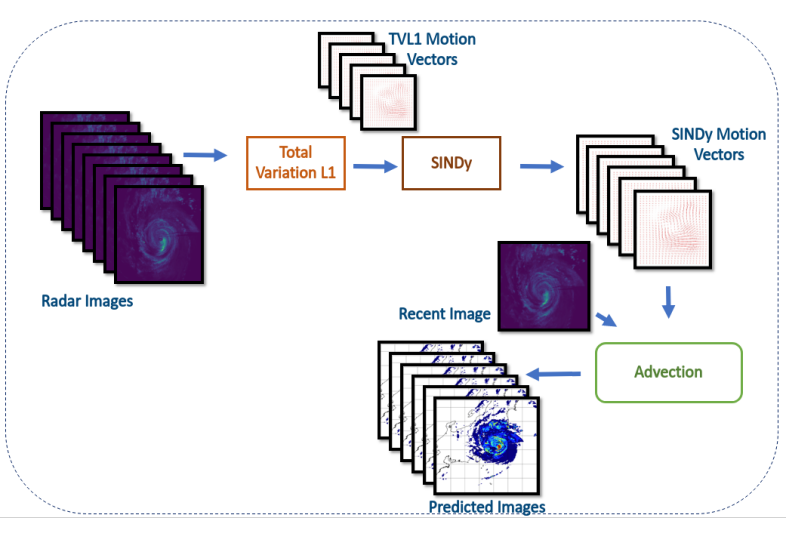

Fig. 2: Proposed TVL1-SINDy Nowcasting System. Here, the motion field is tracked using TVL1-based optical flow which preserves edge details. SINDy captures the non-linear dynamics underlying the time evolution based on a few TVL1 motion vectors. Finally, the SINDy motion field is advected with a recent radar precipitation observation for nowcasting.

the complex non-linear dynamics driving the time evolution of precipitation. Optical flow techniques are widely used for tracking motion from early 1980s. Optical flow estimation techniques with smoothness constraints for optimal solution suffered from inappropriate smoothing of edges and resulted in loss of significant details []. Additionally, optical flow algorithms generally assume rigid surfaces. Hence, we selected Zach et al.'s Total Variation L1 norm-based optical flow method (TVL1) that works with non-rigid surfaces such as precipitation clouds [10]. TVL1 optical flow effectively preserves edges and our initial experiments demonstrated its better performance in capturing the global motion of non-rigid regions.

Nowcasting can be carried out by extrapolating radar reflectivity image with the motion features. But, a mere extrapolation at times fails in capturing the time evolution accurately as the extrapolation scheme alone cannot figure out the underlying non-linear dynamics. Exploring physics models for representing the non-linear dynamics of weather events started earlier [11], [12], [13]. We attempted to use the Sparse Identification of Non-linear dynamics (SINDy), proposed by Brunton et al. in 2016 for depicting the nonlinear time evolution of precipitation [14], [15]. SINDy is a data-driven reduced-order model developed for discovering the governing equations of non-linear dynamical systems from a few samples of previous data. To the best of our knowledge, this is the first work to consider SINDy for representing the non-linear dynamics for precipitation nowcasting.

To summarize, our contributions are:

- A nowcasting system with an edge-preserving tracking method for non-rigid precipitation images using TVL1based optical flow technique.

- An attempt to capture the non-linear dynamics of storm events with the Sparse Identification of Non-linear Dynamics (SINDy)

- Performance evaluation of the proposed TVL1-SINDy nowcasting system revealing the significance of edge details in capturing motion features and the role of nonlinear dynamics in the time evolution of precipitation in nowcasting. The evaluation is conducted on 6 events from Japan Metereology Agency (JMA).

This article is organized as follows. Section 2 discusses prior works on nowcasting based on precipitation features. Section 3 briefly explains the sequential phases comprising a precipitation nowcasting system. The proposed precipitation nowcasting system based on TVL1-SINDy is elaborated in Section 4. Section 5 elaborates on the different experiments for validating the effectiveness of TVL1 on capturing motion field from radar precipitation images, and for evaluating the performance of the proposed TVL1-SINDy system on a set of rainstorm events. Additionally, a detailed discussion of the validation experiments is also included. Finally, Section 6 gives a conclusion of the work highlighting its capabilities and research directions for further improvement.

\section{RELATED WORK}

With the availability of high spatio-temporal resolution radar images, many operational precipitation nowcasting systems with lead times of 1-3 hours were developed including ANC [16], MAPLE [7], STEPS [17] STEPS-BE [18], SWIRLS $[19,20]$.

After the estimation of initial motion vectors from precipitation data, non-linear physics based models were employed [11], [12], [13] to vary the obtained motion vectors over time. Both [11] and [12], used Navier-Stoke's equation to compute the new motion vectors based on the assumption that movement of clouds is a special case of fluid motion. Similarily, Sakaino made use of wave generation theory to estimate future motion vectors [13]. Both these methods demand tuning of several global parameters for different weather scenarios and hence, the better direction will be a data-driven non-linear physics model.

Among the community-driven research and development for nowcasting in the recent years [21], [22], [23], [9], we have used the rainymotion library, an open-source Python library that provides different benchmarking nowcasting models with optical flow methods and extrapolation schemes. There are a few machine learning based nowcasting methods where a model is trained with labelled data and a fixed sequence of precipitation images are generated from the trained model [24], [25], [26]. Machine learning-based models for detection and prediction of weather events require large number of labelled data available in datasets such as 'Digital Typhoon' -the typhoon satellite image dataset by National Institute of Informatics (NII), Japan [27]. The proposed method relies on SINDy - a data-driven model that requires only less number of labelled data for learning a reduced-order representation.

\section{RADAR-BASED PRECIPITATION NOWCASTING}

The features from the precipitation images are tracked for generating motion representation. Tracking involves estimating the apparent motion of precipitation features between two consecutive radar images. Methods estimating the apparent 


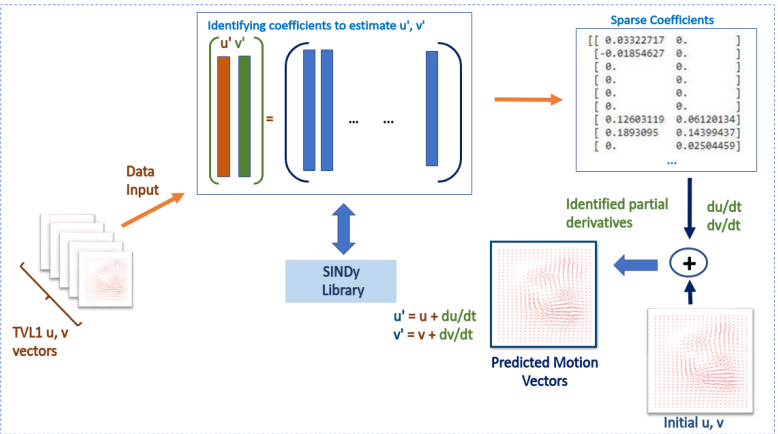

Fig. 3: Schematic diagram of how initial TVL1 motion vectors are understood by SINDy to estimate the partial derivatives $\mathrm{du} / \mathrm{dt}$ and $\mathrm{dv} / \mathrm{dt}$ for generating the motion vector for the next lead time. During training, the model coefficients representing the non-linear dynamics are learned through sparse regression. For prediction, a recent set of motion vectors are given as input to infer $\delta u$ and $\delta v$. Finally, the prediction is generated by adding the $\delta u$ and $\delta v$ with the most recent $\mathrm{u}, \mathrm{v}$ data.

motion from consecutive frames, known as optical flow estimation techniques assume that the information or energy remains constant when a surface is moved to another location with a small displacement. With this assumption, we can have Lagrangian persistence-based schemes for nowcasting. For short lead-time, the Lagrangian persistence-based approach is effective [28], [29]. Many optical flow estimation methods have been developed with the energy constancy assumption such as the seminal works of Lucas-Kanade [30], [31], and Horn-Schunk [32].

\section{Proposed System For Radar-Based PRECIPITATION NOWCASTING}

The performance of a nowcasting system depends on how well it is capable of capturing the motion dynamics of natural phenomena like thunderstorms. In the proposed work, we aimed to improve the nowcasting skill by incorporating an optical flow method that is capable of tracking precipitation features with discontinuities, and by capturing the non-linear dynamics involved in the time evolution of motion features. The Sparse Identification of Non-linear Dynamic (SINDy), proposed by Brunton et al. in 2016 is used for representing the underlying non-linear dynamics [14]. Fig. 2 shows the proposed TVL1-SINDy-based nowcasting system.

\section{A. Total Variation L1 based Tracking of Precipitation}

At pixel level, the optical flow estimation can be defined as a transportation problem, where the intensity is moved to a new pixel location with a small displacement $d x, d y$ while the pixel brightness remains constant. This assumption known as brightness constancy assumption, is represented as,

$$
I(x+d x, y+d y, t+d t)=I(x, y, t)
$$

Here, $I(x, y, t)$ is the pixel intensity at location $(x, y)$ at time $t$. From the location $(x, y)$, the point moves to another location $(x+d x, y+d y)$ as time increases to $t+d t$.
Equation 1 can be rewritten as,

$$
\frac{d}{d t} I(x(t), y(t), t)=0
$$

On applying Taylor serier expansion and using the substitutions $u=\frac{d x}{d t}, v=\frac{d y}{d t}, I_{x}=\frac{\partial I}{\partial x}, I_{y}=\frac{\partial I}{\partial y}$, and $I_{t}=\frac{\partial I}{\partial t}$, we get the Optical Flow Constraint (OFC) as,

$$
I_{x} u+I_{y} v+I_{t}=0
$$

The problem of estimating the two motion vectors, $u$ and $v$ is an ill-posed problem with the two unknowns at each pixel location. Therefore, regularization constraints are introduced such as the smoothness constraint in the Horn-Schunck method. This smoothness constraint results in the loss of sharp edges that are often significant features in image analysis. Most of the optical flow estimation methods are efficient in capturing the motion of rigid body surfaces containing smooth regions. Precipitation images contain non-rigid regions with discontinuities as precipitation clouds grow or decay rapidly [33]. Therefore, we need a robust edge-preserving optical flow estimation method for tracking precipitation features. In the proposed nowcasting system, we used the implementation [34] of Total Variation L1 norm (TVL1) based optical flow estimation scheme devised by Zach et al. [10]. Previously, TVL1 has demonstrated better edge-preserving capability and robustness in image denoising applications [35].

The estimation problem of the motion vector $u$ can be formulated as a minimization of energy functional consisting of the data preservation or the brightness constancy term and the regularization constraint. In TVL1 optical flow, the energy functional is represented as the sum of L1 norm - the data fidelity term and the total variation of $\mathbf{u}$ - the regularization constraint. The energy functional with TVL1 formulation is represented as,

$$
E=\int_{\Omega}\left\{\lambda\left|I_{t}-I_{t+d t}\right|+|\nabla \mathbf{u}|\right\} d x
$$

where $\Omega \subseteq R^{2}, \lambda$ is a free parameter, and $\mathbf{u}=\left[u_{1}, u_{2}\right]^{T}$ represents the motion vector in the $(x, y)$ plane. Equation 4 is computationally infeasible as both the data fidelity term and regularization term are not continuously differentiable. Therefore, Zach et al. followed the duality based minimization scheme proposed by Chambolle [36] that was originally developed for the total variation based image denoising technique proposed by Rudin et al. [35].

Following the derivation of energy minimization in the work of Zach et al. [10], if the image residual is represented as $\rho(u)$ then equation 4 can be rewritten as,

$$
E(u)=\int_{\Omega}\left\{\lambda|\rho(u)|+\left|\nabla u_{1}\right|+\left|\nabla u_{2}\right|\right\}
$$

Applying convex relaxation by introducing an auxiliary variable $v$,

$E_{\theta}(\mathbf{u}, \mathbf{v})=\int_{\Omega}\left\{\lambda|\rho(v)|+\frac{1}{2 \theta}\left|\left(\mathbf{u}-\mathbf{v}^{2}\right)\right|+\left|\nabla u_{1}\right|+\left|\nabla u_{2}\right|\right\}$ 
When $\theta$ is very small, energy becomes minimum when $\mathbf{u}$ and $\mathbf{v}$ are nearly equal. The energy functional can be minimized by alternatively fixing $\mathbf{u}$ or $\mathbf{v}$ and solving for the other variable as discussed in [10].

\section{B. Sparse Identification of Non-linear Dynamics (SINDy)}

SINDy discovers governing equations of nonlinear dynamical systems from data by sparse identification [14]. It is a sparse regression framework by which unknown dynamics of a system can be identified by an extremely sparse brute-force search. Even when the underlying forces are unkown, SINDy identifies the system of governing equations and produce predictions in a purely mathematical manner.

For nowcasting radar precipitation, we used a partial derivative model derived from base SINDy model. SINDy model is initially trained with a few samples of the data, and then used for predicting the precipitation. Fig. 3 illustrates how training a SINDy model is conducted as well as how an inference is obtained from a SINDy model. For training, we used the past ' $\mathrm{n}$ ' motion fields consisting of $u$, $v$ with the spatio-temporal grid. A set of model coefficients for the dynamics is learned on training. Internally SINDy builds a library of all polynomial and derivative combinations up to a prespecified degree $x$ and uses sparse regression to arrive at an approximately linear combination of input and output vectors.

Later, the previously calculated model coefficients are used for predicting the precipitation images. The pre-trained SINDy model estimates the $\delta u$ and $\delta v$, that can be added to the recent $u$ and $v$ to get the next motion vector $u^{\prime}$ and $v^{\prime}$.

$$
\begin{aligned}
& u^{\prime}=u+\delta u \\
& v^{\prime}=v+\delta v^{\prime}
\end{aligned}
$$

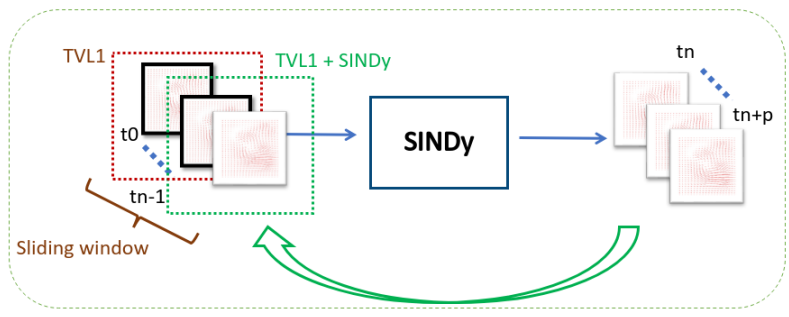

Fig. 4: The sliding window method used while generating SINDy motion vectors. For generating motion vectors at the first step for a given lead time, SINDy uses a set of motion vectors generated by TVL1 optical flow. For the next time step, SINDy takes the newly generated motion field along the TVL1 motion vectors by sliding the window of TVL1 motion fields by one step. The process continues until the motion vectors for the entire lead time is generated.

At each timestep for the specific lead time, SINDy generates a motion field. An advection scheme uses the motion field along with a recent radar image for predicting the precipitation. For a lead time of 1 hour with a time interval of 10 minutes, TVL1 provides the initial set of motion fields.
Subsequent motion fields are provided to SINDy in a sliding window manner. Fig. 4 shows the sliding window method generating SINDy motion fields for the subsequent timesteps.

TABLE I: Characteristics of weather events considered for evaluation.

\begin{tabular}{lllll}
\hline Event No. & Event Name & Date & $\begin{array}{l}\text { Radar Image Size } \\
\text { (height x width) }\end{array}$ & $\begin{array}{l}\text { Geographical Coordinates } \\
\text { (lat 1, lat 2, long 1 long 2) }\end{array}$ \\
\hline 1 & $\begin{array}{l}\text { Typhoon Faxai } \\
\text { Squall lines }\end{array}$ & $\begin{array}{l}\text { 09 August, 2019 } \\
\text { 30 June, 2019 }\end{array}$ & $\begin{array}{l}781 \times 561 \\
721 \times 1201\end{array}$ & $\begin{array}{l}3137127142 \\
32.539136143\end{array}$ \\
3 & Tropical Storm Nari & 26 July, 2019 & $1081 \times 881$ & 3039133144 \\
4 & Typhoon Krosa & 15 August, 2019 & $1561 \times 1201$ & 2841127142 \\
5 & Heavy Rainfall & 25 October, 2019 & $1621 \times 1081$ & 31.545133 .5147 \\
6 & Typhoon Trami & 30 September, 2018 & 1321 x 961 & 3142132144 \\
\hline
\end{tabular}

\section{EXPERIMENTS AND RESULTS}

\section{A. Experimental Setup}

We used the source code of TVL1 implementation proposed by Perez et al. [34]. The TVL1 module needs two recent radar precipitation images that are used for generating a single TVL1 based motion field. The TVL1 implementation requires the parameters such as nproc indicating the number of threads to use in the library, $\tau$ representing the time step used in the numerical scheme, $\lambda$ indicating the weight of the data term in the energy functional, $\theta$ representing the tightness parameter of the minimization surface, nscales for representing the number of scales used in the pyramidal structure, $z$ factor representing the downsample factor for each scale, nwarps representing the number of warps at each scale, $\epsilon$ for the stopping threshold for the iterative optimization. In all the experiments, the parameters nproc, $\tau, \lambda, \theta$, nscales, zfactor, nwarps, $\epsilon$ are set to $0,0.250 .15,0.3,5,0.5,5,0.01$ respectively.

The SINDy model ${ }^{1}$ takes the past motion fields to generate 6 frames for 1-hour long nowcasting with ten-minute interval. For training and prediction, the parameters of the SINDy model such as space derivative order, polynomial degree, sample rate, sparsity cut off, and derivative accuracy are set to $2,2,0.01,0.01,2$ respectively. For advection, the proposed method uses the Constant-Vector (CV) advection scheme available in the rainymotion library [9].

\section{B. Radar Data}

For the proposed TVL1-SINDy nowcasting system, we used the radar precipitation images provided by the Japan Meteorological Association (JMA). Radar mosaic composite reflectivity factor images have a temporal resolution of $10 \mathrm{~min}$ and a spatial resolution of $1 \mathrm{~km}$. The radar precipitation images are used to generate the initial motion field using TVL1. For assessing the nowcasting skills of the proposed method, the radar precipitation observations are used as ground truth.

Table I lists the characteristics of weather events analyzed in this research. Among the events, there are two Typhoons - Faxai, and Krosa, a Squall line, a Heavy Rainfall, and a Tropical Storm named Nari. JMA radar data are used for weather events 1-6.

\footnotetext{
${ }^{1}$ https://github.com/luckystarufo/pySINDy
} 


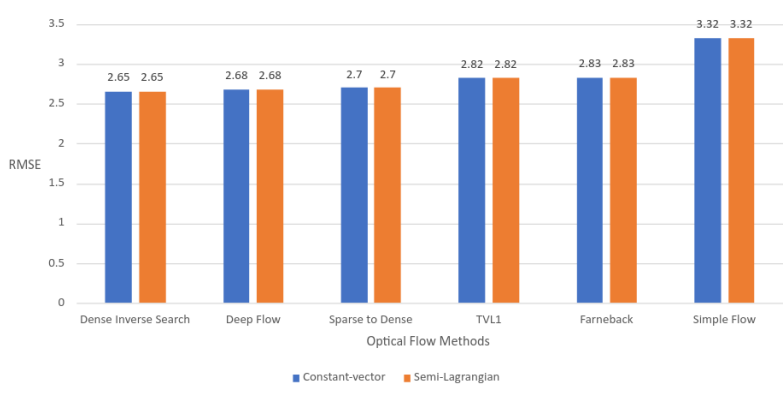

Fig. 5: Comparison of TVL1 optical flow with different optical flow estimation techniques. The optical flow estimation techniques available in OpenCV that can be directly used with rainymotion library are included for the comparison.

\section{Benchmark Models used for Performance Comparison}

For evaluating the performance of the proposed nowcasting model, we used the 5 benchmark models available in the rainymotion library [9] - 2 Sparse Models, 2 Dense Models, and Persistence. Each of the models is briefly explained below.

Sparse Category. In the Sparse category, the motion features are computed from a reduced set of feature points like corner points with a significant gradient in the precipitation intensity. Initially, the feature points are identified using ShiTomasi corner detector [37] and motion features are estimated using the Lucas-Kanade optical flow technique [31]. An affine transformation is used to estimate the pixel locations at each lead time step using the least square estimation for advection. The affine transformation matrix helps to reduce the errors in estimating the feature locations in the advection step. Finally, the feature point at $t$ is warped into the future location at $t+1$ using the affine transformation matrix. There are two models in the Sparse category - The Sparse and Sparse Single Delta.

The Sparse Single Delta uses only 2 previous radar frames. The Sparse model takes 24 previous radar precipitation images for motion feature estimation. Only consistent features from the 24 radar images are considered for further advection. Also, there is a difference in the advection scheme used in the Sparse model. In the Sparse model, an independent linear regression model is used for estimating the time evolution of each feature point.

Dense Category. The two models from the Dense Category - Dense and Dense Rotation consider all the pixels between two radar images for motion estimation. The motion vectors are estimated using Dense Inverse Search (DIS) proposed by Kroeger et al. [38]. The Dense model uses constantvector advection whereas the Dense Rotation model uses semiLagrangian advection capable of capturing rotational motion.

Persistence. Persistence refers to Eulerian persistence based on precipitation persistence where the prediction at $(t+n)^{\text {th }}$ step is considered the same as the radar precipitation observed at $t$.

\section{Performance Evaluation Metrics}

The evaluation of optical flow techniques on the Middlebury dataset is available ${ }^{2}$. As we selected TVL1 in the proposed work based on its prior edge-preserving capabilities demonstrated in applications including image denoising. For evaluating the effectiveness on interpolation of radar precipitation images we used Interpolation Error (IE) [39] as defined in Section V-D1. The performance of the proposed nowcasting schemes is evaluated with respect to the ground truth radar observations from the JMA. The comparison of predicted precipitation images and the ground truth radar observations are carried out at two-level such as the continuous evaluation, and a categorical comparison. For continuous evaluation, Mean Absolute Error (MAE) is used, and for categorical evaluation, the Critical Success Index (CSI) is used. The MAE and CSI are estimated as discussed in Section V-D2 and Section V-D3 respectively.

1) Root Mean Square Error (RMSE): We selected the RMSE metric to measure the effectiveness of TVL1 as precipitation images are generated from a recent radar reflectivity data along with the optical flow vectors. The RMSE is defined as in equation 9

$$
R M S E=\left[\frac{1}{N} \sum_{x, y}\left(I_{P}(x, y)-I_{R}(x, y)\right)^{2}\right]^{\frac{1}{2}}
$$

where $I_{P}$ is the predicted precipitation image, $I_{R}$ is the ground truth radar observation, $(x, y)$ is the pixel location, and $N$ is the total number of pixels.

2) Mean Absolute Error: The Mean Absolute Error (MAE) is defined as the arithmetic average of absolute differences between the ground truth observation and the predicted precipitation image. Equation 10 represents MAE. As MAE represents the error in prediction, a low value indicates better performance.

$$
M A E=\frac{\sum_{x=1}^{M} \sum_{y=1}^{N}\left|I_{P}(x, y)-I_{R}(x, y)\right|}{M * N}
$$

where $I_{P}$ is the predicted precipitation image, and $I_{R}$ is the ground truth radar observation, $(x, y)$ is the pixel location, $M$ is the total number of rows, and $N$ is the total number of columns in the image.

3) Critical Success Index: The categorical performance evaluation metric referred to as the Critical Success Index (CSI) or Threat Score (TS) measures the skill of warning [40]. Equation 11 represents the CSI estimation as a match of pattern between the ground truth radar observation and the predicted precipitation images. CSI indicates the accuracy of prediction by representing the fraction of successful predictions. Hence, higher values indicate better prediction performance.

$$
C S I=\frac{N_{s}}{N_{f}+N_{s}+N_{f a}}
$$

\footnotetext{
${ }^{2}$ http://vision.middlebury.edu/flow/eval/results/results-i1.php
} 
where $N_{s}, N_{f}, N_{f a}$ represents the number of successes, number of failures, number of false alarms at each pixel level respectively.

4) Confidence Interval: Confidence intervals are estimated at each lead step as follows,

$$
C I=\mu \pm z * \frac{s}{\sqrt{n}}
$$

where $\mu$ represents the average value, $z$ is the $z$-score, $s$ is the standard deviation, and $n$ represents the total number of observations. For confidence intervals of $90 \%, 95 \%, 99 \%$, the z-scores are taken as 1.645, 1.960, and 2.576 respectively.

\section{E. Effectiveness of TVL1 Optical Flow}

For evaluating the effectiveness of TVL1 optical flow in tracking the motion vectors, we considered the optical flow techniques that are used in the benchmark models [9]. For advection, both constant-vector and semi-Lagrangian schemes are considered. Both constant vector and semi-Lagrangian gave the same RMSE as the motion features are linear in the immediate next precipitation image, Fig. 5 shows the RMSE obtained for TVL1 and other optical flow techniques.

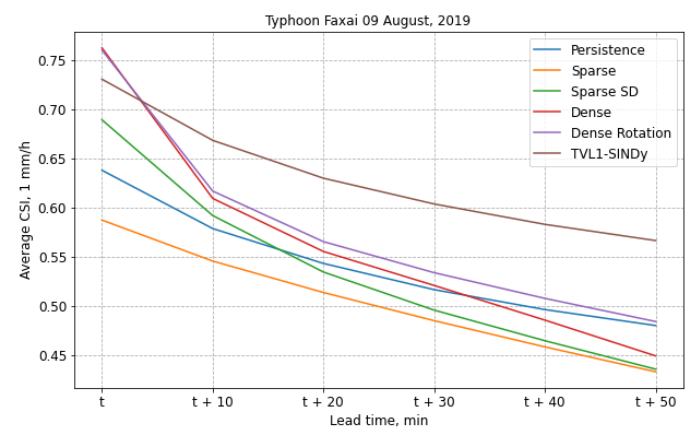

Fig. 6: Comparison of average CSI values obtained for predictions for the event Typhoon Faxai.

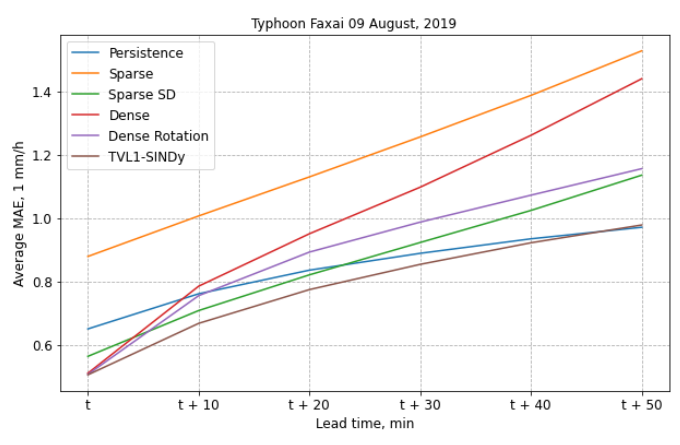

Fig. 7: Comparison of average MAE values obtained for predictions for the event Typhoon Faxai.

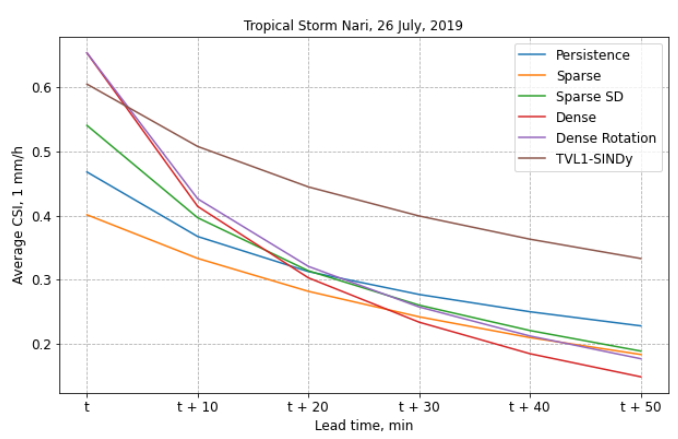

Fig. 8: Comparison of average CSI values obtained for predictions for the event Tropical Storm Nari.

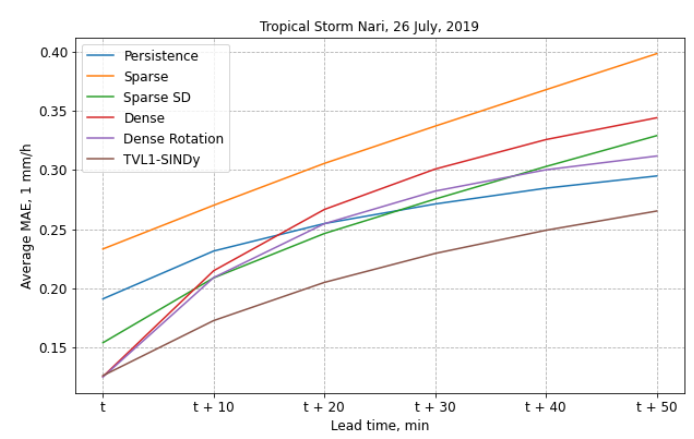

Fig. 9: Comparison of average MAE values obtained for predictions for the event Tropical Storm Nari.

\section{F. Comparison with the Benchmark Models}

We used 6 events listed in Table I with the 5 models from the rainymotion library and the proposed TVL1-SINDy model for performance evaluation. For performance comparison, a total of 109 samples with different initial time are considered for 1-hour nowcasting. The initial lead time is labelled as ' $t$ ', subsequent lead steps with a ten minute interval till ' $t+50$ '.

1) Typhoon Faxai: Fig. 6 shows the average CSI values and Fig. 7 shows the average MAE values obtained for 1-hr nowcasting with ten minute interval for Typhoon Faxai event. In Fig. 6, for the initial lead time, Dense models shows highest average CSI. From the next lead time onwards, the proposed TVL1-SINDy demonstrates the highest average CSI.

In Fig. 7, for the initial lead time, the proposed TVL1SINDy and Dense models shows lowest average MAE. From the next lead time onwards, the proposed TVL1-SINDy demonstrates the lowest average MAE.

2) Tropical Storm Nari: Fig. 8 and Fig. 9 shows the average CSI and average MAE values obtained for the Tropical Storm Nari event respectively. For the initial lead time, Dense models show highest average CSI and TVL1-SINDy shows highest CSI for the subsequent lead times.

In Fig. 9, the proposed TVL1-SINDy shows lowest MAE in all the lead steps. Dense models show the lowest MAE in the initial lead time along with the proposed TVL1-SINDy. 


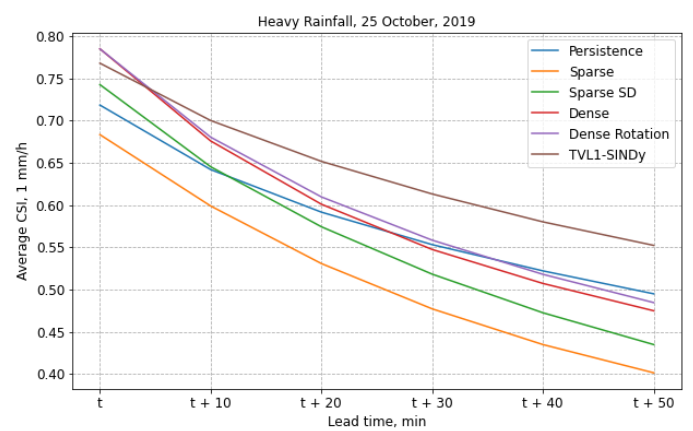

Fig. 10: Comparison of average CSI values obtained for predictions for the event Heavy Rainfall.

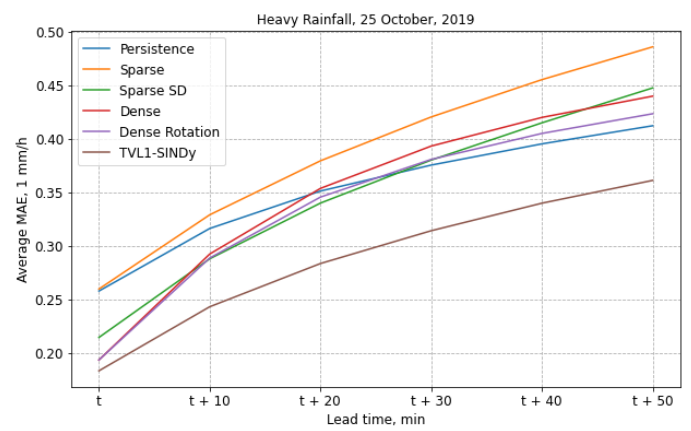

Fig. 11: Comparison of average MAE values obtained for predictions for the event Heavy Rainfall.

3) Heavy Rainfall: Fig. 10 and Fig. 11 shows the average CSI and average MAE values obtained for the Heavy Rainfall event respectively. For the initial lead time, Dense models show highest average CSI and TVL1-SINDy shows highest CSI for the subsequent lead times.

In Fig. 11, the proposed TVL1-SINDy shows lowest MAE in all the lead steps.

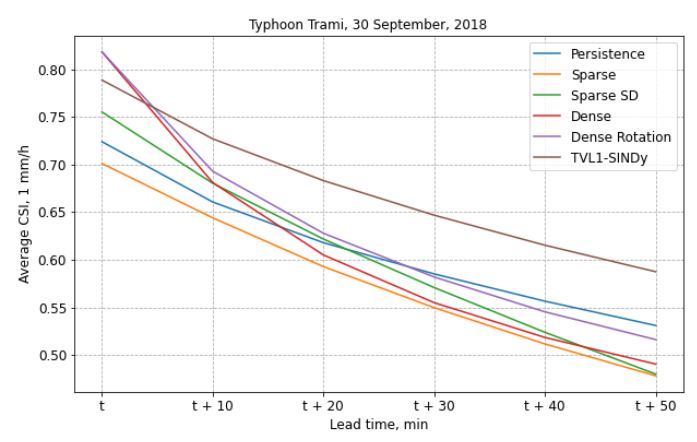

Fig. 12: Comparison of average CSI values obtained for predictions for the event Typhoon Trami.

4) Typhoon Trami: Fig. 12 and Fig. 13 shows the average CSI and average MAE values obtained for the Typhoon Trami respectively. For the initial lead time, Dense models show

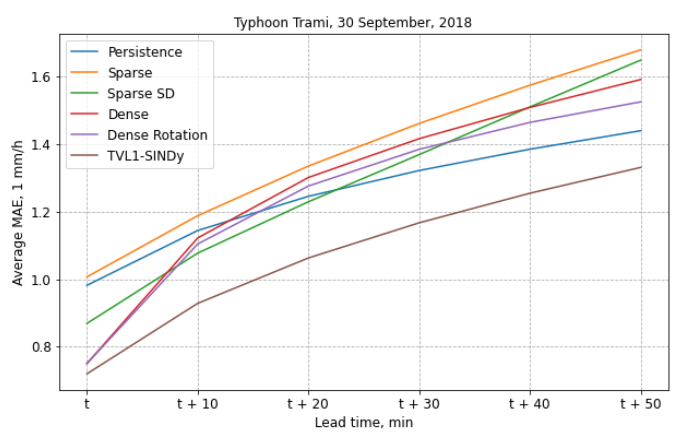

Fig. 13: Comparison of average MAE values obtained for predictions for the event Typhoon Trami.

highest average CSI and TVL1-SINDy shows highest CSI for the subsequent lead times.

In Fig. 13, the proposed TVL1-SINDy shows lowest MAE in all the lead steps.

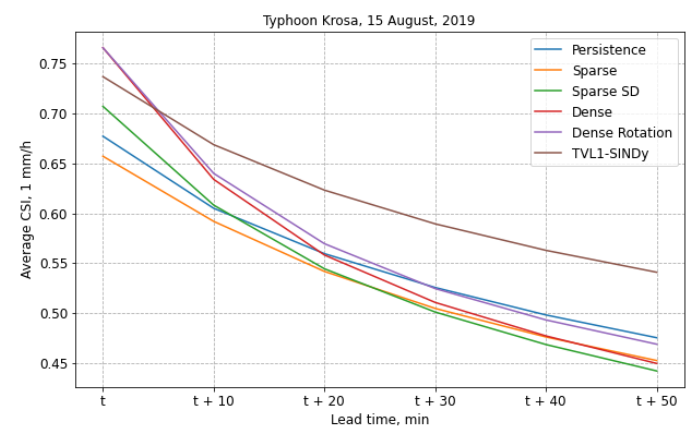

Fig. 14: Comparison of average CSI values obtained for predictions for the event Typhoon Krosa.

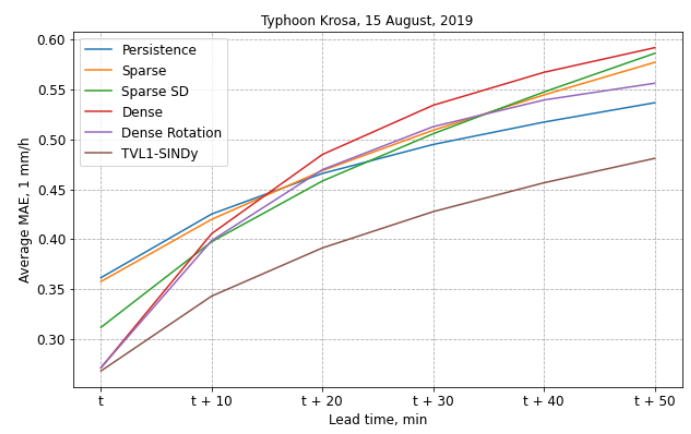

Fig. 15: Comparison of average MAE values obtained for predictions for the event Typhoon Krosa.

5) Typhoon Krosa: Fig. 14 and Fig. 15 shows the average CSI and average MAE values obtained for the Typhoon Krosa respectively. For the initial lead time, Dense models show highest average CSI and TVL1-SINDy shows highest CSI for the subsequent lead times. 
In Fig. 13, the proposed TVL1-SINDy shows lowest MAE in all the lead steps. In the initial lead time, Dense models also demonstrate lowest MAE.

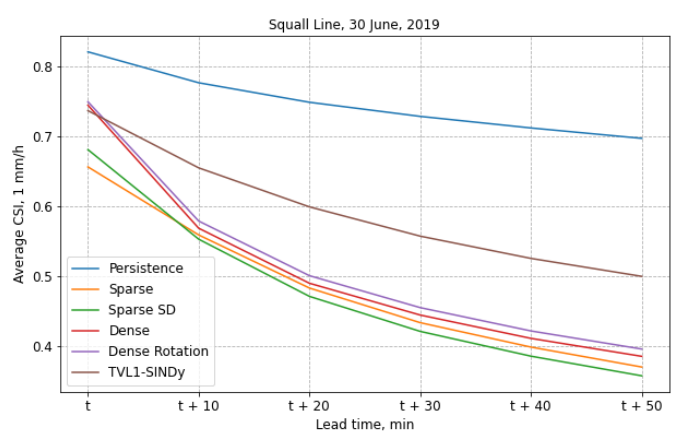

Fig. 16: Comparison of average CSI values obtained for predictions for the Squall Line event.

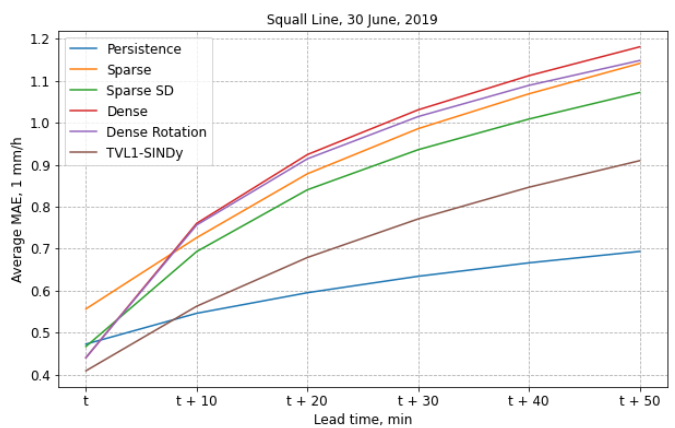

Fig. 17: Comparison of average MAE values obtained for predictions for the Squall Line event.

6) Squall Line: Fig. 16 and Fig. 17 shows the average CSI and average MAE values obtained for the Squall Line event respectively. For this event, Persistence model shows highest average CSI for all lead time. Dense models show second highest CSI for the initial lead time. The proposed TVL1-SINDy shows second highest average CSI from lead time ' $t+10$ ' onwards.

In Fig. 17, the proposed TVL1-SINDy shows lowest average MAE for the initial lead time ' $t$ '. For all the lead times from ' $t+10$ ' onwards, the Persistence model shows second lowest average MAE.

7) Visual Comparison: Fig. 18 shows the nowcasts generated for lead times $t_{0}, t_{0}+10, t_{0}+30, t_{0}+50$ for the Typhoon Faxai event happened on 09 August 2019 at $t_{0}=21: 00$. We included ground truth observations of Typhoon Faxai along with the best performing models from the Sparse and Dense categories in the rainymotion library for a visual comparison of predicted precipitation images. Though predictions of the proposed model look similar to the ground truth than predictions from the Dense Rotation and Sparse SD models, there is an inappropriate elongation in the region around $139.5 \mathrm{E}, 36 \mathrm{~N}$. Fig. 19 shows mountains in this region. In the proposed TVL1SINDy, the non-linear dynamics of the Typhoon is captured based on the motion of precipation alone. The presence of local orographic features have an impact on the time evolution of an event. Considering orographic features with spatiallydependent Boundary Value Problems (BVPs) while capturing the non-linear dynamics can avoid such unrealistic predictions. Further investigation with the SINDy model proposed for Boundary Value Problems - SINDy-BVP is required to consider spatially-dependent features [41].

8) Evaluating Predictions with Threshold set to $5 \mathrm{~mm} / \mathrm{hr}$ : Fig. 20 and Fig. 21 show the evalutaion results obtained for CSI and MAE respectively with a threshold value of $5 \mathrm{~mm}$ per hour for the Typhoon Faxai event. The performance ranking for TVL-SINDy is same as for CSI with threshold $1 \mathrm{~mm}$ per hour. The noticeable difference between the average CSI with threshold of $1 \mathrm{~mm}$ per hour and $5 \mathrm{~mm}$ per hour is that the prediction skill at initial lead time for the proposed TVL1SINDy is same as that of Dense Models for threshold of $5 \mathrm{~mm}$ per hour. Fig. 21 shows lowest average MAE for the proposed model for all the lead times.

9) Evaluating Predictability Limit: The estimation of predictability limit is conducted by a 3-hr prediction of Typhoon Faxai. Fig. 22 and Fig. 23 shows the average CSI, and average MAE values obtained at different lead times. For $3 \mathrm{hr}$ prediction, we took 97 samples with different initial time. Experimental results demonstrate that the CSI is above 0.5 till 2-hr. However, several factors including orographic features, and nature of clouds have to be considered for fixing a predictability limit. From 1 -hr prediction experiments conducted on 7 events, except for Tropical Storm Nari, the CSI stayed above 0.5 for $1 \mathrm{hr}$. Therefore, the predictability limit is fixed at $1 \mathrm{hr}$.

\section{G. Discussion}

Table II shows confidence intervals obtained for 3-hr prediction with threshold $1 \mathrm{~mm}$ per hr for Typhoon Faxai. We can have a confidence of $90 \%, 95 \%, 99 \%$ with $\mu+-0.02$, $\mu+-0.02$, and $\mu+-0.03$ respectively, where $\mu$ represents the average CSI value obtained for that lead step. Table III shows confidence intervals obtained for 1-hr prediction with threshold $5 \mathrm{~mm}$ per hour for Typhoon Faxai. Table III also shows that a confidence of $90 \%, 95 \%, 99 \%$ is achieved with $\mu+-0.02, \mu+-0.02$, and $\mu+-0.03$ respectively.

As expected during the modeling of complex natural phenomena with many known and unknown influencing factors, the nowcasting skill of the proposed model starts to drop off as lead time increases. At the same time, the reliability of nowcasting can be improved by incorporating recent radar images, and recent motion fields for estimating the nowcasts for hours ahead.

From the experiments carried out to reveal the effectiveness of TVL1 in detecting the motion field, it became clear that the constant-vector and semi-Lagrangian schemes in the rainymotion library have no significant difference in advecting the radar image for the immediate lead step. Furthermore, the semi-Lagrangian is said to capture the rotation. Since SINDy in the proposed method captured the non-linear dynamics, we considered constant vector advection when we used the TVL1-SINDy model for generating precipitation images. 

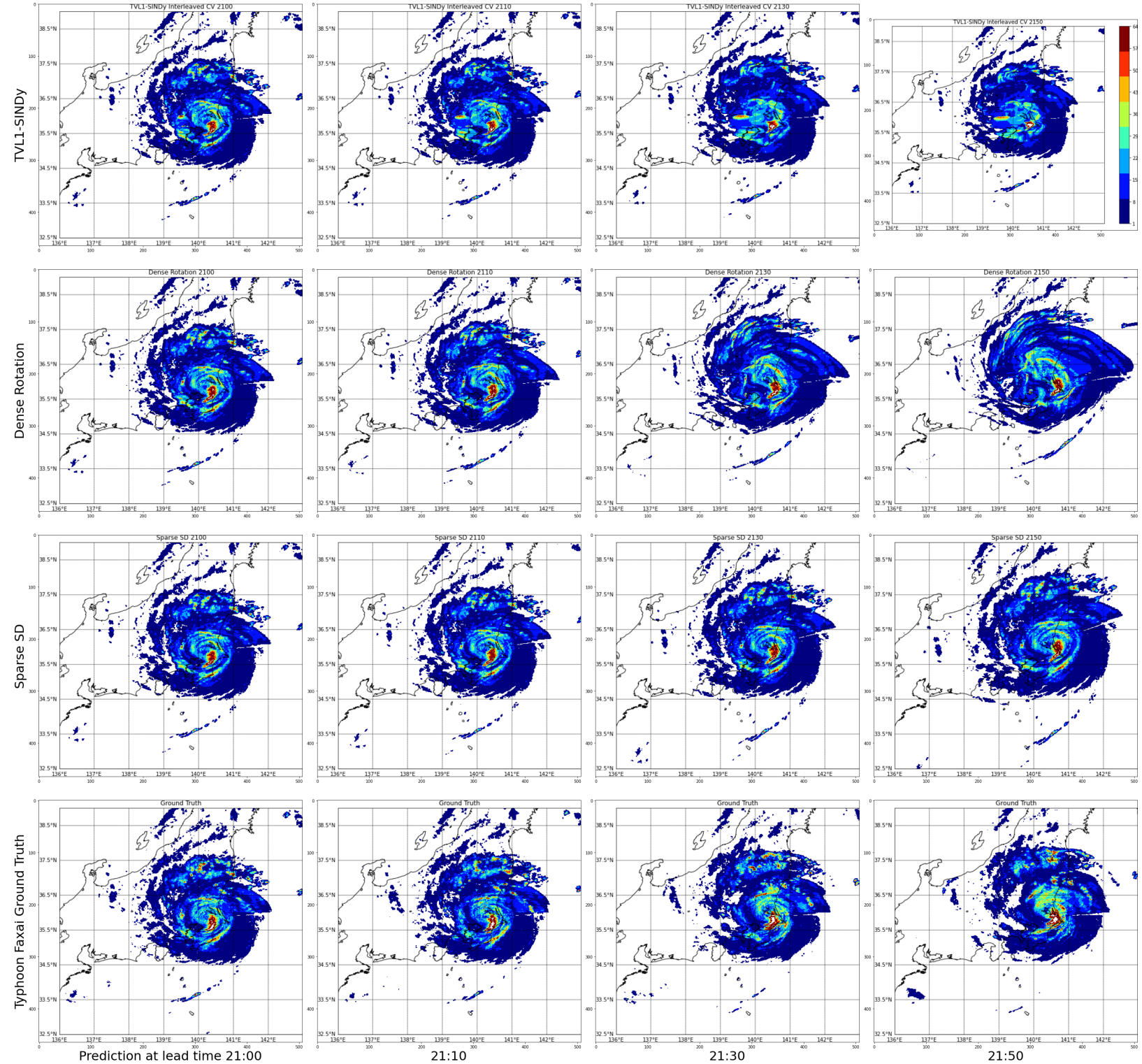

Fig. 18: Example of the nowcasting models outputs for Typhoon Faxai starting at timestep 21:00 on 09 August 2019. The bottom row shows the ground truth. The predicted precipitation images for the proposed model is given in the top row. For comparison, the prediction images generated from the best performing models - SparseSD (second row from the bottom) and Dense Rotation (third row from the bottom) in the Sparse and Dense categories respectively from the rainymotion library are included. To maintain clarity, only the outputs at timesteps 21:00, 21:10, 21:30, and 21:50 are included. The quantized color map represents rainfall in $\mathrm{mm}$ (lowest with dark blue and the highest with red color) as shown in the color chart along with the last output in the first row.

TABLE II: Confidence intervals obtained for CSI with threshold $1 \mathrm{~mm}$ per hr for Typhoon Faxai for 3-hr prediction.

\begin{tabular}{|c|c|c|c|c|c|c|c|c|c|c|c|c|c|c|c|c|c|c|}
\hline $\begin{array}{l}\text { Confidence } \\
\text { interval }\end{array}$ & $t$ & $t+10$ & $t+20$ & $t+30$ & $t+40$ & $t+50$ & $t+60$ & $t+70$ & $t+80$ & $t+90$ & $\mathbf{t}+100$ & $t+110$ & $t+120$ & $t+130$ & $t+140$ & $t+150$ & $t+160$ & $t+170$ \\
\hline $\begin{array}{l}90 \% \% \\
95 \% \% \\
99 \% \%\end{array}$ & $\begin{array}{l}0.73 \pm 0.01 \\
0.73 \pm 0.02 \\
0.73 \pm 0.02\end{array}$ & $\begin{array}{l}0.67 \pm 0.02 \\
0.67 \pm 0.02 \\
0.67 \pm 0.02\end{array}$ & $\begin{array}{l}0.64 \pm 0.02 \\
0.64 \pm 0.02 \\
0.64 \pm 0.03\end{array}$ & $\begin{array}{l}0.61 \pm 0.02 \\
0.61 \pm 0.02 \\
0.61 \pm 0.02\end{array}$ & $\begin{array}{l}0.59 \pm 0.02 \\
0.59 \pm 0.02 \\
0.59 \pm 0.03\end{array}$ & $\begin{array}{l}0.58 \pm 0.02 \\
0.58 \pm 0.02 \\
0.58 \pm 0.02\end{array}$ & $\begin{array}{l}0.56 \pm 0.02 \\
0.56 \pm 0.02 \\
0.56 \pm 0.03\end{array}$ & $\begin{array}{l}0.55 \pm 0.02 \\
0.55 \pm 0.02 \\
0.55 \pm 0.03\end{array}$ & $\begin{array}{l}0.54 \pm 0.02 \\
0.54 \pm 0.02 \\
0.54 \pm 0.03\end{array}$ & $\begin{array}{l}0.53 \pm 0.02 \\
0.53 \pm 0.02 \\
0.53 \pm 0.03\end{array}$ & $\begin{array}{l}0.52 \pm 0.02 \\
0.52 \pm 0.02 \\
0.52 \pm 0.03\end{array}$ & $\begin{array}{l}0.51 \pm .02 \\
0.51 \pm .02 \\
0.51 \pm .03\end{array}$ & $\begin{array}{l}0.50 \pm 0.02 \\
0.50 \pm 0.02 \\
0.50 \pm 0.03\end{array}$ & $\begin{array}{l}0.49 \pm 0.02 \\
0.49 \pm 0.02 \\
0.49 \pm 0.03\end{array}$ & $\begin{array}{l}0.48 \pm 0.02 \\
0.48 \pm 0.02 \\
0.48 \pm 0.03\end{array}$ & $\begin{array}{l}0.46 \pm 0.02 \\
0.46 \pm 0.02 \\
0.46 \pm 0.03\end{array}$ & $\begin{array}{l}0.47 \pm 0.02 \\
0.47 \pm 0.02 \\
0.47 \pm 0.03\end{array}$ & $\begin{array}{l}0.46 \pm 0.02 \\
0.46 \pm 0.02 \\
0.46 \pm 0.03\end{array}$ \\
\hline
\end{tabular}

\section{CONCLUSION}

In the proposed method, we used TVL1-based optical flow technique for estimating the apparent motion of precipitation from two successive images. Subsequently, the data-driven reduced-order SINDy based nowcasting method learns the underlying dynamics responsible for the time evolution of precipitation. TVL1 optical flow is used for tracking the motion field that is used as input for the SINDy method as TVL1 performed well in capturing fine details from the precipitation images. Given a set of a few samples of past motion 


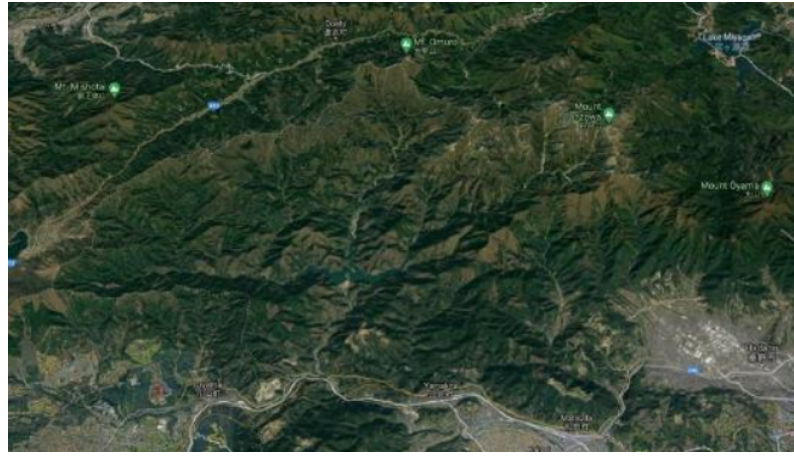

Fig. 19: The geographical region around $(139.5 \mathrm{E}, 36 \mathrm{~N})$ indicating mountains.

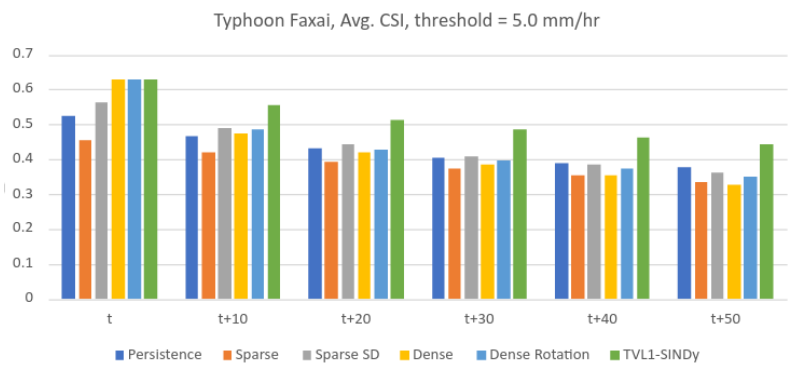

Fig. 20: Comparison of average CSI values obtained for predictions for the event Typhoon Faxai at different initial time with a threshold of $5 \mathrm{~mm} / \mathrm{hr}$.

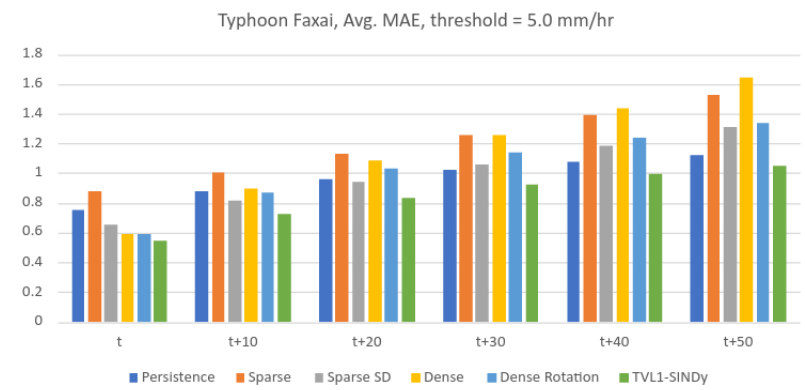

Fig. 21: Comparison of average MAE values obtained for predictions for the event Typhoon Faxai at different initial time with a threshold of $5 \mathrm{~mm} / \mathrm{hr}$.

TABLE III: Confidence intervals obtained for CSI with threshold $5 \mathrm{~mm}$ per hr for Typhoon Faxai.

\begin{tabular}{lllllll}
\hline $\begin{array}{l}\text { Confidence } \\
\text { interval }\end{array}$ & $\mathrm{t}$ & $\mathrm{t}+10$ & $\mathrm{t}+20$ & $\mathrm{t}+30$ & $\mathrm{t}+40$ & $\mathrm{t}+50$ \\
\hline & & & & & & \\
$90 \%$ & $0.63 \pm 0.02$ & $0.56 \pm 0.02$ & $0.51 \pm 0.02$ & $0.48 \pm 0.02$ & $0.46 \pm 0.02$ & $0.44 \pm 0.02$ \\
$95 \%$ & $0.63 \pm 0.02$ & $0.56 \pm 0.02$ & $0.51 \pm 0.02$ & $0.48 \pm 0.02$ & $0.46 \pm 0.02$ & $0.44 \pm 0.02$ \\
$99 \%$ & $0.63 \pm 0.03$ & $0.56 \pm 0.03$ & $0.51 \pm 0.03$ & $0.48 \pm 0.03$ & $0.46 \pm 0.03$ & $0.44 \pm 0.03$
\end{tabular}

fields from previous precipitation images, SINDy is capable of capturing known and unknown influences of a weather event. The better nowcasting skill attributed to the proposed TVL1SINDy method is evident from the experiments conducted on the representative events.

As a training set, SINDy requires only a few sample of past data. By few samples, we mean past 5-10 radar reflectivity images. We need to explore further to know about the effect of a specific number of past samples on the prediction skill. Also, more studies are needed to evaluate the performance of recent optical flow techniques on non-rigid surfaces with significant discontinuities.

One of the significant advantages of the data-driven reduced-order SINDy representation is that SINDy requires only a few numbers of past samples for learning the nonlinear dynamics whereas data-driven machine learning models require a large amount of data for training. SINDy captures the time evolution of numerical data through sparse regression. Based on previous samples, it captures the underlying governing equations for the time evolution. Physics based models captures time evolution dynamics based on known facts, wheras SINDy is a data-driven model that implicity captures the dynamics by generating a library containing a variety of polynomials during the training phase. As future work, we plan to improve the function approximation of SINDy by designing a customized libarary of functions incorporating prior knowledge of causes and forces. The effectiveness of SINDy representation depends on the library of function it generates on training. Therefore, SINDy is able to represent any force or causes that are present in the training set.

We believe that the inclusion of SINDy as a technique to capture non-linear dynamics of the time evolution of rain precipitation would open up new directions for improving the nowcasting skill.

\section{ACKNOWLEDGMENT}

Authors would like to acknowledge Weather News Inc. for sharing the data of seven events comprising of Typhoons and Storms.

\section{REFERENCES}

[1] K. A. Browning. Review lecture: Local weather forecasting. Proceedings of the royal society of London. A. Mathematical and Physical Sciences, 371(1745):179-211, 1980.

[2] B. P. Shukla, P. K. Pal, and P. C. Joshi. Extrapolation of sequence of geostationary satellite images for weather nowcasting. IEEE Geoscience and Remote Sensing Letters, 8(2):216-219, 2010.

[3] B. P. Shukla, C. M. Kishtawal, and P. K. Pal. Prediction of satellite image sequence for weather nowcasting using cluster-based spatiotemporal regression. IEEE transactions on geoscience and remote sensing, 52(7):4155-4160, 2013.

[4] M. Berenguer, M. Surcel, I. Zawadzki, M. Xue, and F. Kong. The diurnal cycle of precipitation from continental radar mosaics and numerical weather prediction models. part ii: Intercomparison among numerical models and with nowcasting. Monthly weather review, 140(8):2689-2705, 2012.

[5] D. G. Jensen, C. Petersen, and M. R. Rasmussen. Assimilation of radar-based nowcast into a hirlam nwp model. Meteorological Applications, 22(3):485-494, 2015.

[6] C. Lin, S. Vasić, A. Kilambi, B. Turner, and I. Zawadzki. Precipitation forecast skill of numerical weather prediction models and radar nowcasts. Geophysical research letters, 32 (14), 2005.

[7] U. Germann and I. Zawadzki. Scale-dependence of the predictability of precipitation from continental radar images. part i: Description of the methodology. Monthly Weather Review, 130(12):2859-2873, 2002. 
Typhoon Faxai $3 \mathrm{hr}$ Prediction Avg. CSI, threshold 1mm/hr

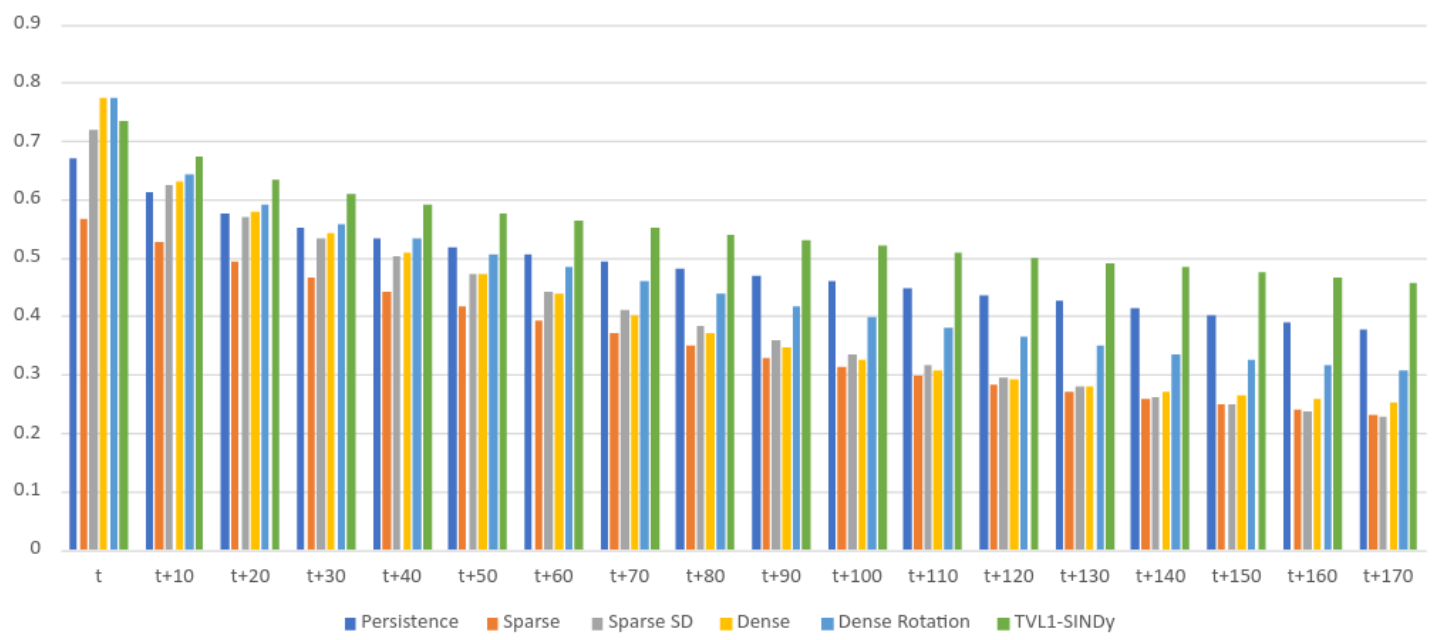

Fig. 22: Comparison of average CSI obtained for predictions generated for $3 \mathrm{hr}$ duration.

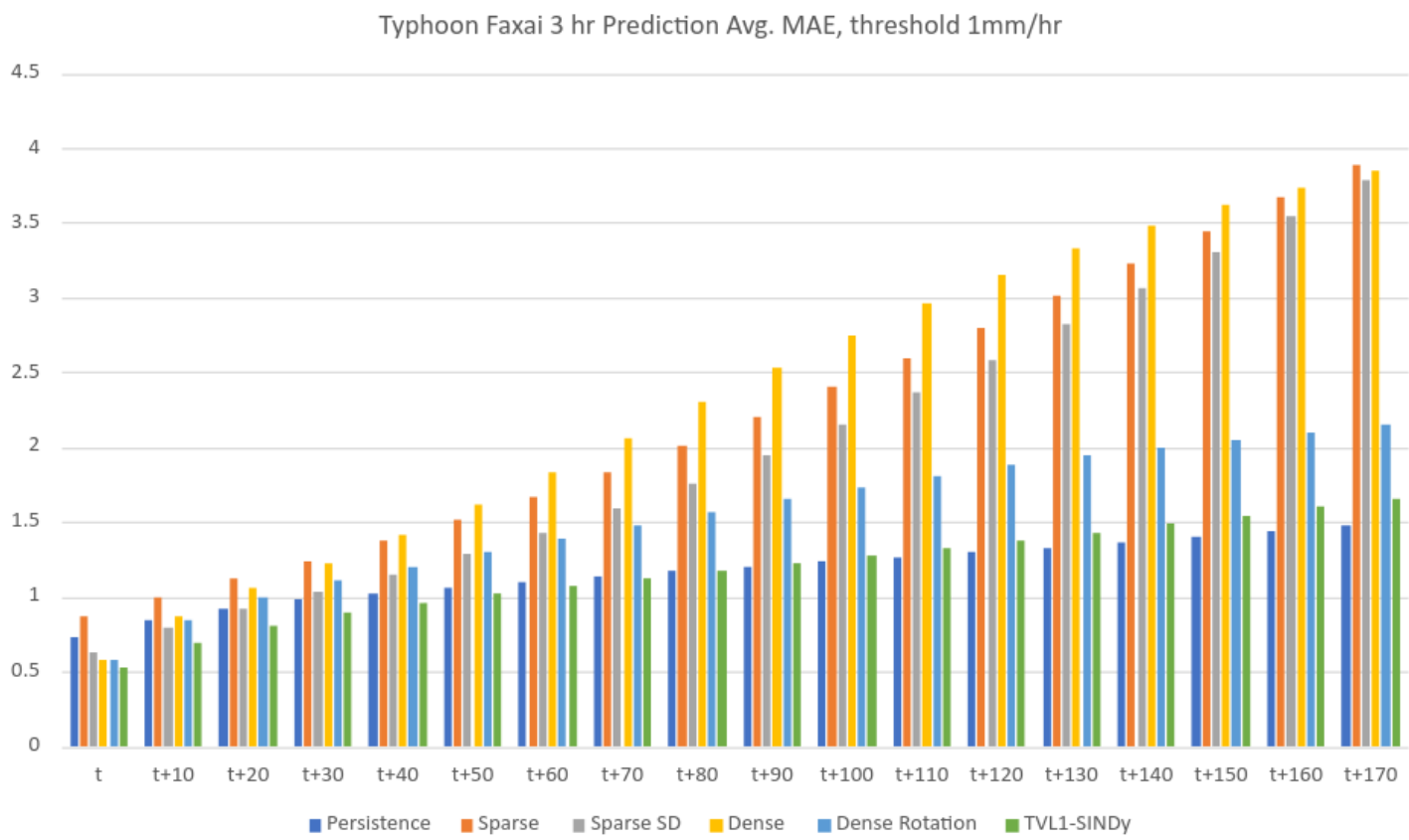

Fig. 23: Comparison of average MAE obtained for predictions generated for $3 \mathrm{hr}$ duration.

[8] M. Reyniers. Quantitative precipitation forecasts based on radar observations: Principles, algorithms and operational systems. Institut Royal Météorologique de Belgique Brussel, Belgium, 2008.

[9] G. Ayzel, M. Heistermann, and T. Winterrath. Optical flow models as an open benchmark for radar-based precipitation nowcasting (rainymotion v0. 1). Geoscientific Model Development, 12(4), 2019.

[10] C. Zach, T. Pock, and H. Bischof. A duality based approach for realtime tv-1 1 optical flow. In Joint pattern recognition symposium, pages 214-223. Springer, 2007.

[11] H. Sakaino. Spatio-temporal image pattern prediction method based on a physical model with time-varying optical flow. IEEE transactions on geoscience and remote sensing, 51(5):30233036, 2012.

[12] K. Mounika, G. Kutty, and S. S. RK. et al. Gorthi. Consistent robust and recursive estimation of atmospheric motion vectors from satellite images. IEEE Transactions on Geoscience and Remote Sensing, 57(3):1538-1544, 2018.

[13] H. Sakaino. Motion estimation for dynamic texture videos based on locally and globally varying models. IEEE Transactions on Image Processing, 24(11):3609-3623, 2015.

[14] S. L. Brunton, J. L. Proctor, and J. N. Kutz. Discovering governing equations from data by sparse identification of nonlinear dynamical systems. Proceedings of the national academy of sciences, 113(15):3932-3937, 2016.

[15] Brian de Silva, Kathleen Champion, Markus Quade, JeanChristophe Loiseau, J. Kutz, and Steven Brunton. Pysindy: A python package for the sparse identification of nonlinear dynamical systems from data. Journal of Open Source Software, 5(49):2104, 2020. doi: 10.21105/joss.02104. URL https://doi.org/10.21105/joss.02104. 
[16] C. Mueller, T. Saxen, R. Roberts, J. Wilson, T. Betancourt, S. Dettling, N. Oien, and J. Yee. Ncar auto-nowcast system. Weather and Forecasting, 18(4):545-561, 2003.

[17] N. E. Bowler, C. E. Pierce, and A. W. Seed. Steps: A probabilistic precipitation forecasting scheme which merges an extrapolation nowcast with downscaled nwp. Quarterly Journal of the Royal Meteorological Society: A journal of the atmospheric sciences, applied meteorology and physical oceanography, 132(620):2127-2155, 2006.

[18] L. Foresti, M. Reyniers, A. Seed, and L. Delobbe. Development and verification of a real-time stochastic precipitation nowcasting system for urban hydrology in belgium. Hydrology and Earth System Sciences, 20(1):505, 2016.

[19] P. Cheung and HY. Yeung. Application of optical-flow technique to significant convection nowcast for terminal areas in hong kong. In The 3rd WMO International Symposium on Nowcasting and Very Short-Range Forecasting (WSN12), pages 6-10, 2012.

[20] W. Woo and W. Wong. Operational application of optical flow techniques to radar-based rainfall nowcasting. Atmosphere, 8 (3):48, 2017.

[21] WK. Wong, VTL. Cheng, and WC. Woo. Community swirls nowcasting system (com-swirls). In WMO WWRP 4th International Symposium on Nowcasting and Very-short-range Forecast (WSN16), page 113, 2016.

[22] T. Haiden, A. Kann, C. Wittmann, G. Pistotnik, B. Bica, and C. Gruber. The integrated nowcasting through comprehensive analysis (inca) system and its validation over the eastern alpine region. Weather and Forecasting, 26(2):166-183, 2011.

[23] S. Pulkkinen, D. Nerini, A. A. Pérez H., C. Velasco-Forero, A. Seed, U. Germann, and L. Foresti. Pysteps: an open-source python library for probabilistic precipitation nowcasting (v1.0). Geoscientific Model Development, 12(10):4185-4219, 2019.

[24] S. Xingjian, Z. Chen, H. Wang, D. Yeung, W. Wong, and W. Woo. Convolutional lstm network: A machine learning approach for precipitation nowcasting. In Advances in neural information processing systems, pages 802-810, 2015.

[25] L. Foresti, I. V. Sideris, D. Nerini, L. Beusch, and U. Germann. Using a 10-year radar archive for nowcasting precipitation growth and decay: A probabilistic machine learning approach. Weather and Forecasting, 34(5):1547-1569, 2019.

[26] G. Ayzel, T. Scheffer, and M. Heistermann. Rainnet v1. 0: a convolutional neural network for radar-based precipitation nowcasting. Geoscientific Model Development, 13(6):26312644,2020

[27] Lucas Rodés-Guirao. Deep learning for digital typhoon: Exploring a typhoon satellite image dataset using deep learning, 2019.

[28] Urs Germann, Isztar Zawadzki, and Barry Turner. Predictability of precipitation from continental radar images. part iv: Limits to prediction. Journal of the Atmospheric Sciences, 63(8):20922108, 2006.

[29] E. Ruzanski and V. Chandrasekar. Scale filtering for improved nowcasting performance in a high-resolution $\mathrm{x}$-band radar network. IEEE transactions on geoscience and remote sensing, 49 (6):2296-2307, 2011.

[30] B. D. Lucas and T. Kanade. Optical navigation by the method of differences. In IJCAI, pages 981-984. Citeseer, 1985.

[31] B. D Lucas and T. et al. Kanade. An iterative image registration technique with an application to stereo vision. 1981.

[32] B. KP. Horn and B. G Schunck. Determining optical flow. In Techniques and Applications of Image Understanding, volume 281, pages 319-331. International Society for Optics and Photonics, 1981.

[33] N. EH. Bowler, C. E Pierce, and A. Seed. Development of a precipitation nowcasting algorithm based upon optical flow techniques. Journal of Hydrology, 288(1-2):74-91, 2004.

[34] J. S. Pérez, E. Meinhardt-Llopis, and G. Facciolo. Tv-11 optical flow estimation. Image Processing On Line, 2013:137-150, 2013.

[35] L. I. R., S. Osher, and E. Fatemi. Nonlinear total variation based noise removal algorithms. Physica D: nonlinear phenomena, 60 (1-4):259-268, 1992.

[36] A. Chambolle. An algorithm for total variation minimization and applications. Journal of Mathematical imaging and vision, 20(1-2):89-97, 2004.

[37] J. et al. Shi. Good features to track. In 1994 Proceedings of IEEE conference on computer vision and pattern recognition, pages 593-600. IEEE, 1994.

[38] T. Kroeger, R. Timofte, D. Dai, and L. Van Gool. Fast optical flow using dense inverse search. In European Conference on Computer Vision, pages 471-488. Springer, 2016.

[39] S. Baker, D. Scharstein, JP. Lewis, S. Roth, M. J. Black, and R. Szeliski. A database and evaluation methodology for optical flow. International journal of computer vision, 92(1):1-31, 2011

[40] J. T. Schaefer. The critical success index as an indicator of warning skill. Weather and forecasting, 5(4):570-575, 1990.

[41] Daniel E Shea, Steven L Brunton, and Nathan Kutz. Sindy-bvp: Sparse identification of nonlinear dynamics for boundary value problems. arXiv preprint arXiv:2005.10756, 2020.

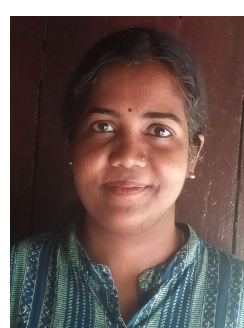

tation Nowcasting.
Divya S. Vidyadharan is a Research Engineer at Augsense Lab, India. She received Ph.D. in Computer Science and Engineering from the University of Kerala in 2019. She received her M.Tech degree in Computer Science with a specialization in Digital Image Computing, from the Department of Computer Science, University of Kerala in 2004 and B.Tech degree in Computer Science and Engineering from SCT College of Engineering, Kerala, India, in 2000. Her research interests include Computer Vision, Digital Image Forensics, and Radar Precipi-

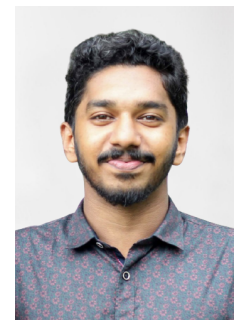

Aaron Xavier received his B.Tech Degree in Electrical and Electronics Engineering from the College of Engineering-Trivandrum. He works as a Research Engineer at Augsense Lab, India. His areas of research are Computer Vision and Robotics.

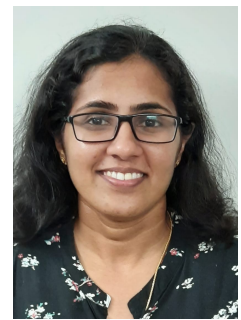

Blossom Treesa Bastian is a Research Engineer at Augsense Lab, India. She received Ph.D. in Electronics and Communication from the University of Kerala in 2020. She received her B.Tech. degree in Electronics and Communication from Cochin University of Science and Technology, Kerala, India, in 2009 and M.Tech degree in signal processing from College of Engineering Trivandrum, Kerala, India, in 2012. Her research interests include Numerical Weather Prediction Models, Computer Vision and Machine Learning and its application in the field of 


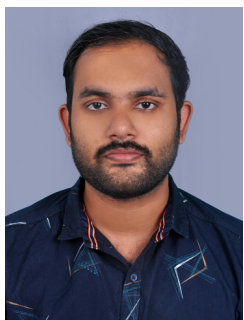

Ajay Ragh received the B.Tech. degree in Electronics and Communication Engineering from Government Engineering College Barton Hill, Trivandrum, Kerala, India. He works as Research Engineer at Augsense Lab, India. His areas of research are Deep Learning, Nowcasting, Nanoelectronics, Computer Vision and Robotics.

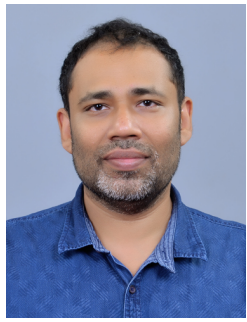

Naveen Francis Chittilapilly is a Research Engineer at Autonomous Control System Lab Ltd, Japan and is a co-founder of Augsense Lab and heads the research activities there. His research interests include Inverse Problems and Machine intelligence, Computational Imaging, Computer Vision with applications in Robotics, Medical Imaging, Remote Sensing. 